\title{
Four Limb Shaped Auricular Chondrocutaneous Composite Graft for the Alar and the Columellar Defects
}

\author{
Seok Min Yoon ${ }^{1}$, Da Woon Lee ${ }^{2}$, \\ Seung Min $\mathrm{Nam}^{3}$, Syeo Young Wee, \\ Hyun Gyo Jeong ${ }^{1}$, \\ Chang Yong Choi ${ }^{1}$ \\ ${ }^{1}$ Department of Plastic and \\ Reconstructive Surgery, Soonchunhyang \\ Gumi Hospital, Gumi; ${ }^{2}$ Department of \\ Plastic and Reconstructive Surgery, \\ Soonchunhyang Seoul Hospital, \\ Soonchunhyang University College of \\ Medicine, Seoul; ${ }^{3}$ Department of Plastic \\ and Reconstructive Surgery, \\ Soonchunhyang Bucheon Hospital, \\ Bucheon, Korea
}

This work was supported by the Soonchunhyang University Research Fund.

No potential conflict of interest relevant to this article was reported.
Background Composite grafts have advantages for small nasal defect coverage. However, if the the outer skin defect and the inner skin defect have a different location, conventional composite grafts encounter considerable limitations. Therefore, we devised a 4-limbed graft to overcome this limitation by use of soft tissue transposition. Methods Over the course of 5 years, this auricular composite graft was used in 10 cases of reconstruction. We harvested skin and cartilage from the helix. The composite graft had 2 limbs of soft tissue to cover the nasal defect and another 2 limbs of cartilage to support nasal framework. The cartilage limbs extended 3 to $5 \mathrm{~mm}$ beyond the margin of the skin. The direction of each limb was modified according to defect position.

Results All 10 composite grafts survived completely. All composite grafts shrank by a small percentage of their bulk. Nonetheless, the nasal framework was maintained to an acceptable extent because of the cartilage limbs.

Conclusions This technique was capable not only of covering defects in the alar and columellar area, but also of maintaining a satisfactory external appearance, because the 2 limbs of cartilage included in the graft strengthened the nasal framework and provided modest support to the nostril margin. The 2 limbs of soft tissue covered the defect area. Our 4-limbed auricular chondrocutaneous composite graft is reliable option for the reconstruction of alar and columellar defects in a single-stage procedure.

Keywords Cartilage, Helix, Reconstructive surgical procedures

\section{INTRODUCTION}

The nose has a dominant position on the face, and is of great importance in the context of facial aesthetics [1]. If trauma, tumor extirpation, or scar contracture causes defect or notching in the nose, aesthetic and functional problems occur as a result. Therefore, the reconstruction of nasal defects is a challenging and important area for plastic surgeons.

Received: Jul 11, 2017 Revised: Sep 24, 2017 Accepted: Sep 25, 2017 Correspondence: Chang Yong Choi Department of Plastic and Reconstructive Surgery, Soonchunhyang Gumi Hospital, Soonchunhyang University College of Medicine, 179 1Gongdan-ro, Gumi 39371, Korea. E-mail: 73120@schmc.ac.kr

Copyright (C) 2017 The Korean Society for Aesthetic Plastic Surgery.

This is an Open Access article distributed under the terms of the Creative Commons Attribution Non-Commercial License (http://creativecommons.org/licenses/by-nc/4.0/) which permits unrestricted non-commercial use, distribution, and reproduction in any medium, provided the original work is properly cited. www.e-aaps.org
Reconstruction is not easy if defects occur in the lower third of the nose, because the lower third of the nose is a single subunit and has limited mobility [2]. Furthermore, the ala has a complex 3-dimensional (3D) shape and a convex curvature with 3 different components (inner mucosa, cartilage supporting the $3 \mathrm{D}$ shape, and outer skin) [3]. For this reason, many factors must be considered when reconstructing a nose, and if an incorrect reconstructive method is chosen, the free margin of the ala can be easily disrupted by imprecise closures [2].

Many methods have been developed for covering nasal defects. The most basic method is primary closure, but this method encounters limitations because alar asymmetry is unavoidable due to the intrinsic tissue defects. In addition, forehead flaps have been developed. However, forehead flaps are likewise not suitable for relatively young patients because they involve a multi-stage technique that is inconvenient for the patient. Local or regional flaps have the disadvantage of causing visible midfacial scars [4]. Finally, free flaps are not used in initial reconstructions, because the proce- 
dure is too invasive compared to other techniques and the longterm results are unsatisfactory. Furthermore, free flaps do not have the delicate 3-layer structure (2 skin layers and 1 cartilage layer) of the nostril lining [3].

Auricular chondrocutaneous composite grafts are relatively commonly used for reconstructing nasal defects. The helix, antihelix, concha, and lobule are often used as the donor sites [3]. The use of an auricular chondrocutaneous composite graft was first described by Brown and Cannon [5] in 1946. The auricular chondrocutaneous composite graft is one of the best methods for nasal defect reconstruction because it allows the reconstruction of the structural framework (i.e., the cartilage) along with reconstruction of the inner mucosa and outer skin within a single stage, and because the surgical time is shorter than is required for other methods. In addition, the auricular composite graft is anatomically similar to the nostril floor because it is composed of 2 skin layers and 1 cartilage layer located between the 2 skin layers [6]. Additionally, the color and texture of the auricular skin are very similar to those of the nose. Furthermore, auricular composite grafts rarely shrink and provide excellent support, as they have similar thickness and stability to the upper and lower lateral cartilage and the fibrocartilaginous complex of the nose. Finally, donor site morbidity is very low due to the ample blood supply of the ear [7]. Composite grafts are an easy technique for covering alar rim and columellar defects, because the ear helix has a relatively convex structure [7]. The helical rim has a thin skin envelope on both sides [8], and the helical margin arc has a convex curvature stabilized by the curved cartilage skeleton. It can be applied to almost any part of the alar margin [8].

For the above reasons, the chondrocutaneous composite graft began to be harvested from the ear helix. However, all grafts have some degree of shrinkage, and even slight shrinkage can cause prominent aesthetic effects on the nose. For this reason, Qing et al. [6] devised a modified composite graft, in which the auricular cartilage is 2 to $3 \mathrm{~mm}$ wider than the auricular skin border so that the cartilage can be easily inserted into the nostril recipient tissue and can strengthen the nasal framework, minimizing the scar contracture that may occur after graft shrinkage [6].

Soft-triangle defects on the nose are more difficult to correct than dorsal defects. This is because the soft triangle may have different defect directions in the inner mucosa and outer skin when defects occur. For example, the inner mucosa can form a defect vector of 3 oclock, while the outer skin may have a defect vector of 9 oclock. To overcome these problems, a 4 -limbed ear helix chondrocutaneous composite graft was proposed. Our study reports an improvement of the previous auricular composite graft. The 4 -limbed composite graft consisted of 2 soft-tissue limbs and 2 cartilage limbs. The 2 soft-tissue limbs were intended to cover the soft-tissue defect of the nostril lining, and the 2 cartilage limbs were intended to cover the nasal framework. This structure may be able to effectively cover the nostril defect with a convex curvature and with different layers for the inner mucosa and outer skin.

Therefore, a composite graft with 4 limbs was devised, with 2 limbs (the soft-tissue/cartilage portion) cross-linked with each other. This technique adequately covered the nostril defect site. The purpose of this article is to introduce a series of patients in whom a 4-limbed auricular chondrocutaneous composite graft was used to reconstruct the defects of the ala and soft triangle.

\section{METHODS}

Ten patients who underwent nasal reconstruction using a chondrocutaneous composite graft were analyzed. The indications for the chondrocutaneous composite graft included notching or defect of the ala and stricture of the nostril. Their preoperative and postoperative photographs, office charts, and hospital records were reviewed retrospectively.

\section{Donor site considerations and harvesting}

The helical rim and root were used as donor sites for all grafts. To make each defect site normal, we analyzed the size, shape, contour and components of the defect site after releasing of the scar. A similar portion of the ear was used for the graft, and was harvested. When the incision line was considered, the helical crus of the ear was used to retain the cartilaginous portion of the graft beyond the margins of the overlying skin graft to serve as stabilizing limbs once the graft was inserted in the recipient site (an "inter-locking graft") [9].

The incision line of the donor site was marked on either side, following the curvature of the helical crus. The donor site was anesthetized with $1 \%$ lidocaine with diluted epinephrine. The donor site was incised using a No. 15 blade, and cutting was done perpendicular to the posterior surface of the auricular skin. Composite grafts are more often harvested from the anterior surface of the auricular concha than from the posterior surface [9]. However, when harvesting the helical cartilage in this study, the posterior surface of the helical skin was chosen, because the volume of the skin and the subcutaneous soft tissue of the posterior surface is thicker than that of the anterior surface. Graft tissues frequently shrink in terms of width and thickness [10]. In order to address this concern, we designed the composite grafts to be larger than the estimated defect preoperatively. The horizontal length of the soft tissue was 7 to $8 \mathrm{~mm}$ and the vertical length was approximately $15 \mathrm{~mm}$. Thus, we obtained enough soft tissue to maintain the nostril floor. The cartilage limbs were then harvested. The cartilage extended 3 to $5 \mathrm{~mm}$ beyond the margin of the skin portion (Fig. 1). Both sides of the skin harvested with cartilage were dissected $5 \mathrm{~mm}$ from the cartilage using Metzenbaum scissors. This was done to separate the cartilage limb and the soft-tissue limb, thereby making the soft-tissue limb transposable according to the defect shape. Once excised, the graft was placed on a cold sterile saline-soaked gauze. 
The donor site was closed with simple interrupted sutures. Primary closure of the donor site was achieved for a defect less than $15 \mathrm{~mm}$ as described by Singh and Bartlett [11] in 2007.

The composite graft was inserted into the soft-triangle defect in

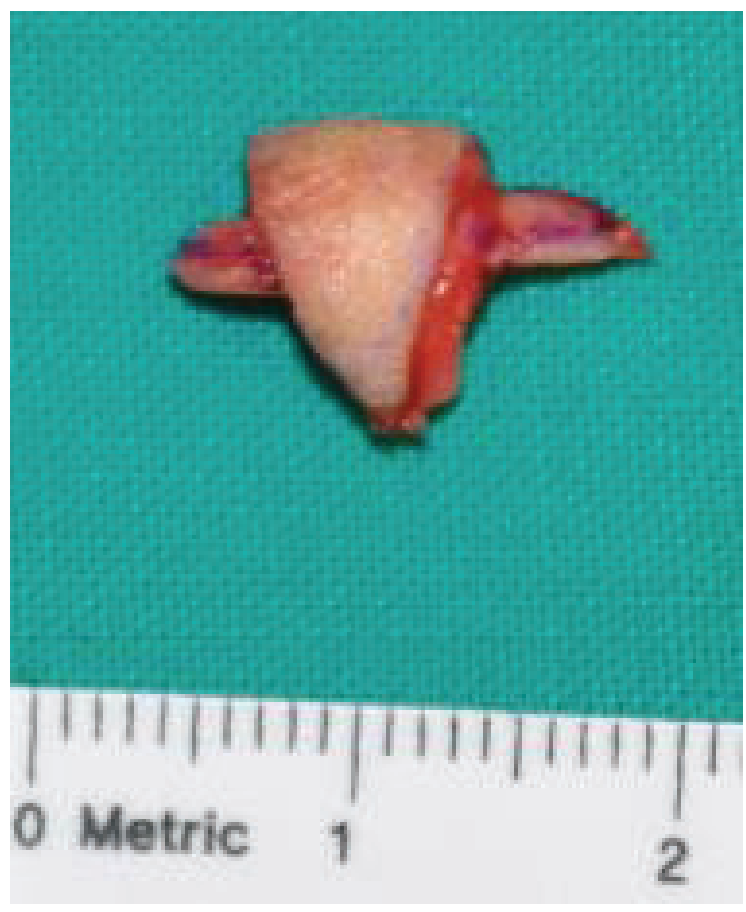

Fig. 1. Cartilage harvested from the helical rim had a horizontal length of about $15 \mathrm{~mm}$ and a vertical length of about 7 to $8 \mathrm{~mm}$. We obtained 2 limbs of proper soft tissue and 2 limbs of cartilage.

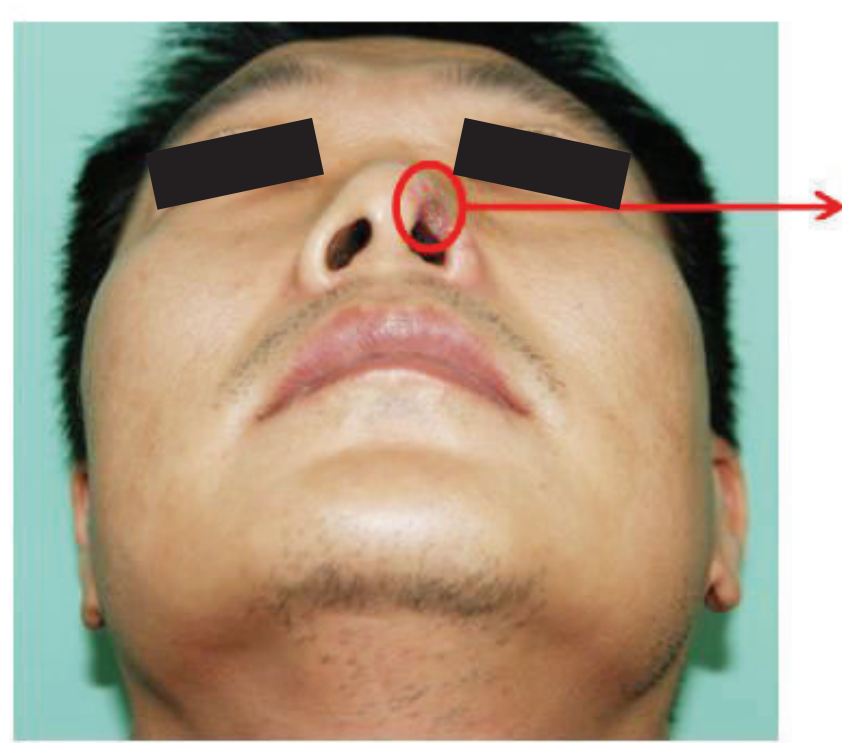

the nostril area. The 2 cartilage limbs were inserted in the nostril lining (Fig. 2). A proper nasal lining was obtained from the 2 cartilage limbs of the composite graft. After the insertion of the cartilage, the defect area was covered by transposing the skin portion attached to the cartilage. The direction of the soft-tissue limb was modified according to the position of the defect (e.g., columella, vestibule, or ala). Therefore, the remaining 2 limbs of soft tissue were able to be flexibly transposed at the angle necessary to cover the defect of the soft triangle 3D (Fig. 2).

\section{Suturing the graft}

We took care to preserve the vascular components, to remove all devitalized tissue, and to increase the contact surface not only of the wound edge, but also of the wound bed. Using curved Metzenbaum scissors, the skin was trimmed to expose the underlying cartilage 3 to $5 \mathrm{~mm}$ beyond the margin of the skin portion. The 2 remaining soft-tissue limbs were used to cover the nostril soft-tissue defect. Once the cartilage had interlocked, the external skin of the graft was approximated using No. 6-0 non-absorbable sutures. To minimize vessel strangulation and to enhance the anastomoses between the 2 vascular beds, subcutaneous tissue suturing was not performed. The cartilage itself was not sutured on any nasal surface. After the suture, a wet dressing was placed on the graft site to improve the success rate, using an antibiotic ointment but not using an immobilized compression (e.g., Merocele ${ }^{\circledast}$ packing). We did not use any foam dressing or occlusive dressing. The patients did not use an extra pillow because the soft-tissue limb of the composite graft could maintain the appropriate nostril lining. Additionally, if the graft is compressed by a pillow, it may interfere with skin graft

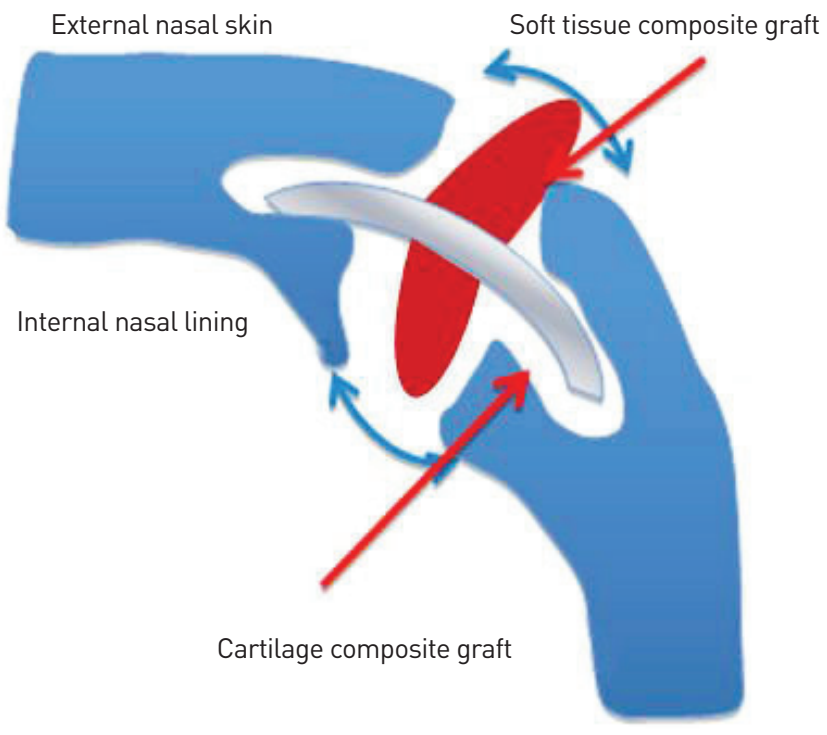

Fig. 2. The 2 limbs composed of soft tissue (red portion) could be flexibly transposed at the desired angle and could cover the defect of the soft triangle 3-dimensionally. 
success by affecting the vascular circulation.

\section{Postoperative care}

After the operation, the graft site manifested swelling and a reddish skin color. Then, within 1 to 2 days, the color of the graft became cyanotic purple due to venous congestion, which remained approximately until week 2 , until adequate venous drainage developed. The stitches were removed after 15 days. The patients visited the hospital every month for postoperative monitoring.

\section{RESULTS}

Over a 5-year period, 10 patients underwent nasal reconstruction using the chondrocutaneous composite graft. All 10 grafts completely survived, and none of the patients showed a noticeable contour deformity on the donor site. Throughout the long-term follow-up period, however, all the composite grafts shrank by a small percentage, and the patients presented varied degrees of pigmentation. Nonetheless, the shrinkage was minimized by the 3 to $5 \mathrm{~mm}$ extension of the cartilage over the skin, and we performed laser treatment to correct discoloration.

Thus, satisfactory results were obtained from the nostril reconstruction procedures, and deformities of the donor sites of large defects were minimized via the 2-limb soft-tissue portion and the other 2-limb cartilage portion of the composite graft from the ear and open ointment moisture balance dressing. Two typical cases are presented below.

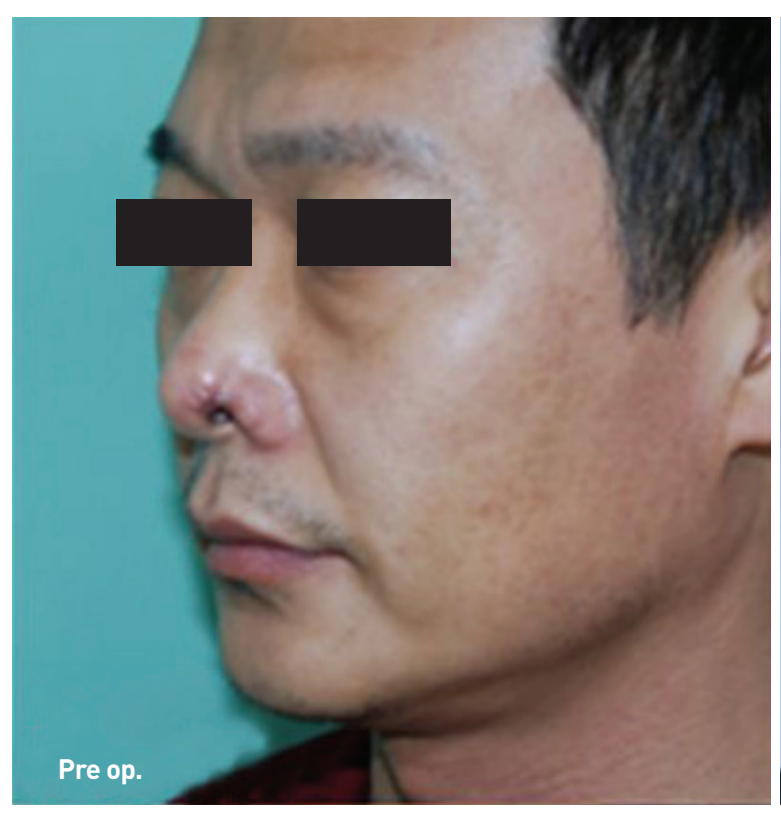

\section{Case 1}

A 43-year-old man was referred to our plastic surgery unit due to a human bite. On examination, the patient exhibited a definite defect (approximately $1 \times 0.5 \mathrm{~cm}$ ) in the soft triangle of the left alar area, with a collapsed left-nostril lining (Fig. 3). For the donor site, the middle helix of the ipsilateral ear was targeted. A temporary template was created using foam dressing material, and the composite

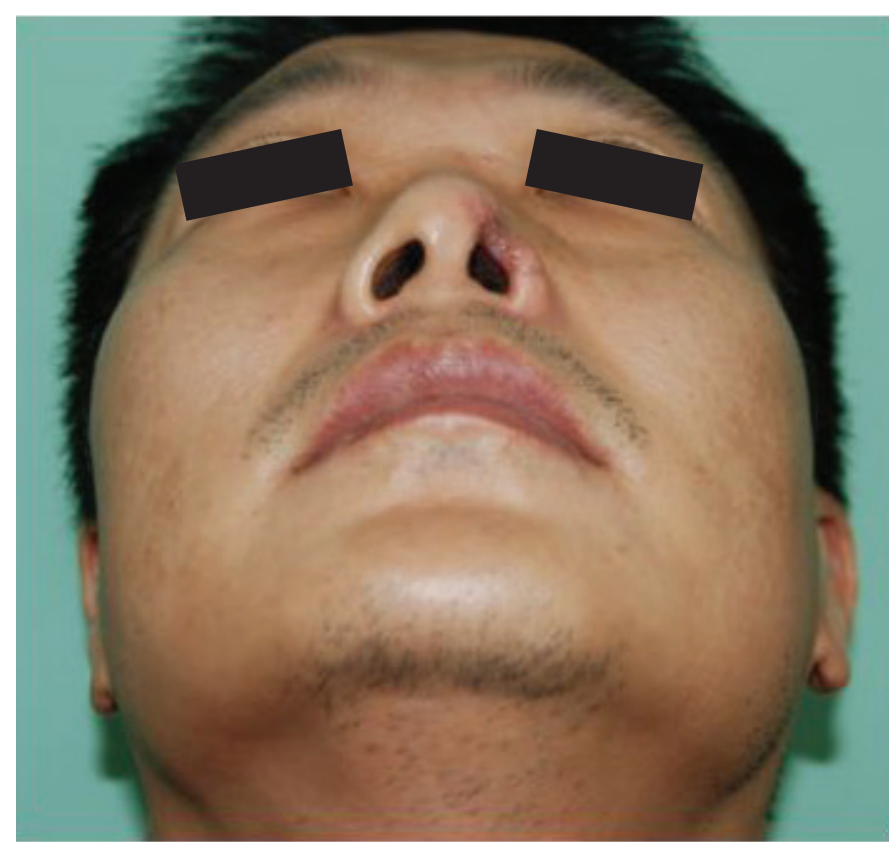

Fig. 3. Preoperative photographic findings of case 1: a worm's eye view.

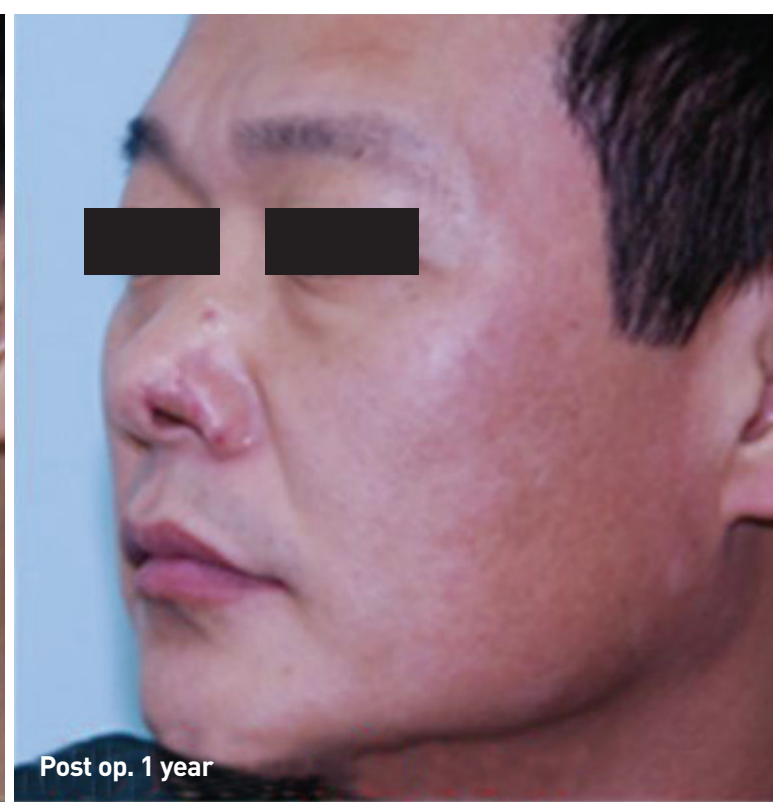

Fig. 4. Change of the left nostril lining in an oblique view. During the follow-up period, complete coverage of the defect with excellent wound healing was obtained, with good results from the functional and aesthetic point of aureus. OP, operation. 

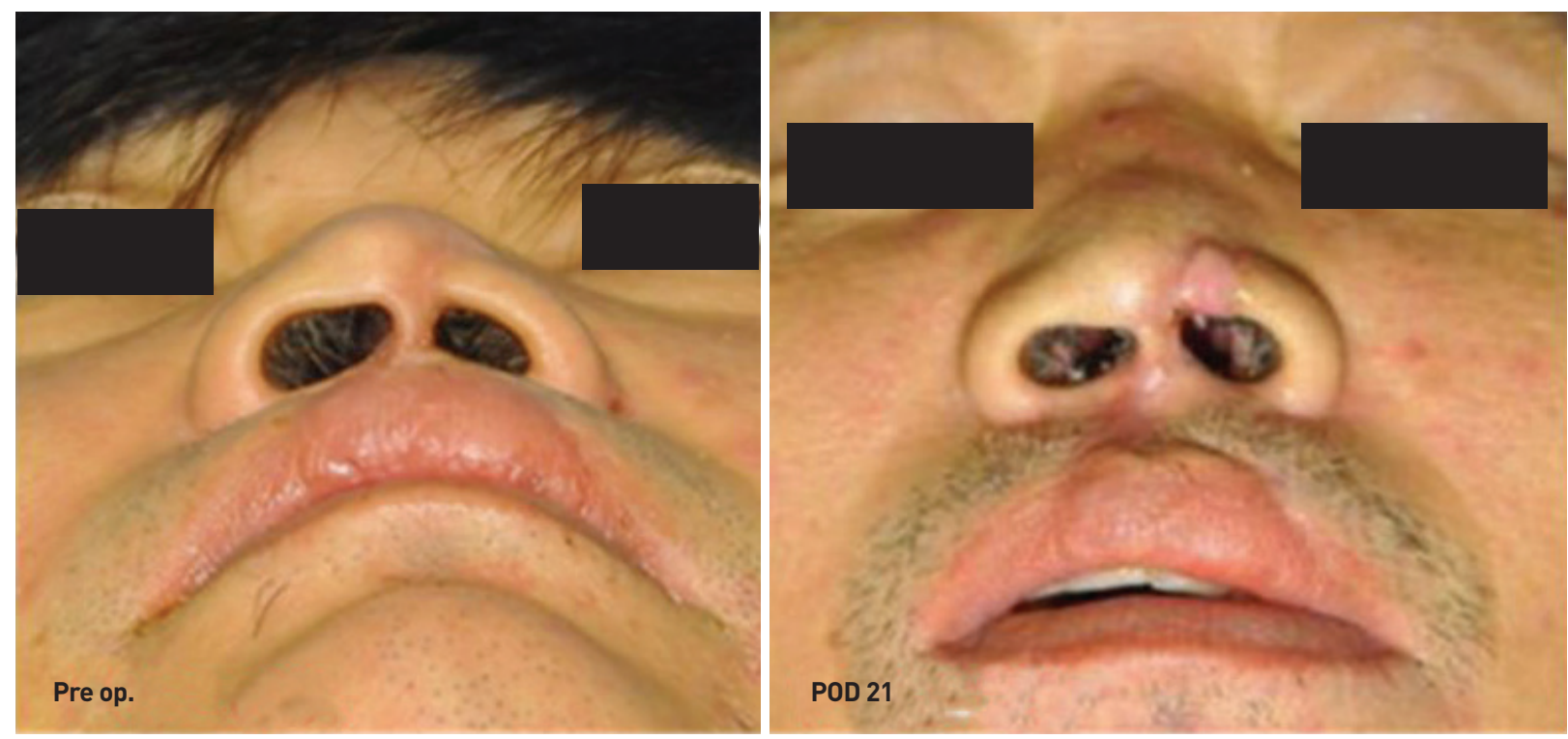

Fig. 5. Change of left nostril lining in a worm's eye view during the follow-up period. OP, operation; POD, post operative days.

graft was gently harvested from the ipsilateral auricular middle helix area.

After meticulous dissection of the graft recipient site, minimal debridement was carefully performed at the recipient site, and the composite graft was sutured at the nostril lining.

After the operation and the serial follow-up, complete coverage of the defect with excellent wound healing results was obtained from both the aesthetic and functional viewpoints (Fig. 4).

\section{Case 2}

A 53-year-old man had a soft-tissue triangle defect at the anterior nostril site and a columellar vestibule defect at the posterior nostril site due to a traffic accident. Additionally, he had an asymmetric nostril lining, with the right part larger than the left part (Fig. 5). We used a temporary foam template, as in case 1 , and harvested the composite graft. The same 4 -limbed cartilage composite graft as in case 1 was produced, and the composite graft was inserted. After the operation, the nostril asymmetry was corrected, an acceptable range of contour deformity was present on the outer side of the nostril, and no other complications occurred (Fig. 5). As a result, the patient was subjectively satisfied with the surgical outcome.

\section{DISCUSSION}

Koreans have smaller noses than Caucasians [12]. As such, composite graft procedures are suitable for ethnic Koreans and others with a similarly narrow ala and columella. These proportions require a complex and delicate operative technique, as the composite graft has a flexible structure and can have small components.
The difference between our operation and the conventional composite graft is that our method involved 2 soft-tissue limbs and 2 cartilage limbs. It is also noteworthy that each of the 2 limbs had a 4-limb structure that intersected with that of the other. In particular, the 2 soft-tissue limbs contained a certain portion for forming a transposition flap, which was useful for covering defects of the alar and columellar areas that had various vector directions. Due to the flexibility of the soft-tissue portion, a transposition flap with a rotation arc was possible. As the rotation arc functioned independently in the 2 soft-tissue limbs, the inner and outer surfaces of the nostril could be covered in different anatomical areas (Fig. 4). We were also able to cover even larger defects because the soft tissue limb could flexibly cover the defect area. Previous studies have suggested that composite grafts are not a suitable method for covering large defects. For example, Brown and Cannon [5] concluded that a graft greater than $1 \mathrm{~cm}$ is not suitable for reconstruction because of limited blood flow, resulting in subsequent graft necrosis. However, in this study, the soft tissue limb intersected with the cartilage limb, and the flap-like shape was used to transpose the soft tissue limb to widen the contact surface with the recipient bed. For this reason, not only was it possible to cover defects larger than $1 \mathrm{~cm}$, but the graft success rate also increased.

As this graft contained 2 cartilage limbs with a solid character, there was no need for an inner retainer to hold the nostril or device in place to compress it. This promoted the patients' comfort. Moreover, the cartilage limbs were useful for strengthening the nasal framework, minimizing potential future scar contracture, and maintaining the nostril shape. Finally, as 2 layers of skin were harvested from the donor site, the full-layer nostril defect could be reconstructed in a single stage, making the procedure convenient, cost-effec- 
tive, and less invasive.

Nonetheless, this study has several potential limitations. First, composite grafts are not frequently used in large defects because of the reduced survival rate [1]. Adams and Ratner [13] stated that the use of composite grafts is limited for large defects $(>2 \mathrm{~cm})$ due to their limited blood flow. Composite grafts need relatively more metabolites than simple skin grafts, and require rapid revascularization to be successful [13]. The revascularization of a composite graft starts in the subdermal plexus of the graft and the recipient donor bed [13]. However, this circulation is limited in terms of its ability to extend beyond the margin of the composite graft [13]. Any part of the graft that is more than $1 \mathrm{~cm}$ from these vessel anastomoses does not have a sufficient blood supply, hindering complete graft success [13]. Additionally, the survival of the cartilage of the composite graft depends on the passive diffusion of oxygen and of the nutrients of the perichondrium from around the well-vascularized tissue [13]. Therefore, vascularity is more important in composite grafts, and as such, the restriction of the length is more absolute. Thus, grafts wider than $2 \mathrm{~cm}$ are at risk for central ecchymotic necrosis [13]. Second, there is a risk of cartilage infection because auricular cartilage is vulnerable to postoperative infection [2]. In fact, prophylactic antibiotics were used to prevent infection of the recipient cartilage and the auricular donor suture site. Another limitation is the contour deformity and pigmentation due to scarring between the soft tissue of the recipient site and the composite graft. This is inevitable, but can be treated by laser treatment or a fat graft.

\section{CONCLUSION}

The 4-limbed auricular chondrocutaneous composite graft is a reliable option for the reconstruction of alar and columellar defects in a single stage. It is a simple and superior method for reconstructing the ala and columella compared to other operations in terms of color, shape, flexibility, and structural support. Relatively large alar and columellar defects can be reconstructed successfully with this composite graft, without donor site morbidity.

\section{PATIENT CONSENT}

Patients provided written consent for the use of their images.

\section{REFERENCES}

1. Ferreira S, Ayres Quaresma LE, Timoteo CA, et al. The primary closure approach of dog bite injuries of the nose. J Craniofac Surg 2014; 25:e216-8.

2. Klinger M, Maione L, Villani F, et al. Reconstruction of a full-thickness alar wound using an auricular conchal composite graft. Can J Plast Surg 2010;18:149-51.

3. Son D, Kwak M, Yun S, et al. Large auricular chondrocutaneous composite graft for nasal alar and columellar reconstruction. Arch Plast Surg 2012;39:323-8.

4. Teltzrow T, Arens A, Schwipper V. One-stage reconstruction of nasal defects: evaluation of the use of modified auricular composite grafts. Facial Plast Surg 2011;27:243-8.

5. Brown JB, Cannon B. Composite free grafts of two surfaces of skin and cartilage from the ear. Ann Surg 1946;124:1101-7.

6. Qing Y, Cen Y, Chen J, et al. Reconstruction of a large through-andthrough defect of the nasal tip using a modified auricular composite graft. J Craniofac Surg 2015;26:382-3.

7. Haug MD, Rieger UM, Witt P, et al. Managing the ear as a donor site for composite graft in nasal reconstruction: update on technical refinements and donor site morbidity in 110 cases. Ann Plast Surg 2009; 63:171-5.

8. Peach AH, Stretch JR. Improved selection and management of the auricular composite graft donor site. Br J Plast Surg 2004;57:797-9.

9. Ratner D, Katz A, Grande DJ. An interlocking auricular composite graft. Dermatol Surg 1995;21:789-92.

10. Rossell-Perry P, Romero-Narvaez C. Evaluation of the use of auricular composite graft for secondary unilateral cleft lip nasal alar deformity repair. Plast Surg Int 2014;2014:270285.

11. Singh DJ, Bartlett SP. Aesthetic management of the ear as a donor site. Plast Reconstr Surg 2007;120:899-908; discussion 9.

12. Kwon HJ, Han KH, Kim JH, et al. Photogrammetry based on standardized clinical photography using cephalostat: Comparison with anthropometric analysis. J Korean Soc Plast Reconstr Surg 2007;34:24-36.

13. Adams C, Ratner D. Composite and free cartilage grafting. Dermatol Clin 2005;23:129-40, vii. 